\title{
Nachtrag zur Pathogenese der sympathischen Ophthalmie, III.
}

\author{
Von \\ Prof. R. Deutschmann in Göttingen.
}

Die Beobachtung, die hier als Nachtrag zu meinem obigen Aufsatze ihren Platz findet, hätte eigentlich ihrer grossen Wichtigkeit nach, die sie für die von mir entwickelte Anschauung hat, in den Mittelpunkt meiner Beweisführung, wenigstens für den fraglichen Process beim Menschen, gestellt werden müssen. Indess, wie das oft so geht, führte mir der Zufall dieselbe erst $\mathrm{zu}$, als die obige Arbeit längst abgeschlossen und gedruckt war. So innig ist aber das, was ich noch mittheilen möchte, mit dem oben Erörterten verknüpft, so sehr wesentlich unterstützt es, ja beweist es die von mir vertretenen Ansichten grade für die sympathisehe Ophthalmie beim Menschen, dass ich mich für verpflichtet hielt, es wenn auch nur im Anschluss an diese, so doch jedenfalls noch vorzubringen.

Fink, 15 Jahre, aus Holzerode kam am 18. Juli 1884 zur Augenklinik; gestern Abend sei ihm, als er bei Steineklopfern auf der Chaussee vorüberging, ein Stück Stein gegen das rechte Auge geflogen, wonach sich sofort das Sehvermögen verschlechterte. Status präsens: R. Auge mässig injicirt, Lappenwunde der Hornhaut nach i. o. mit Irisanlegung, kleines Iricolobom nach i. 0.; Cataract in Quellung. Augendruck normal. 
Lichtschein präcis, Projection unsicher. Keine Erscheinung einer Infection mit Sicherheit, deshalb Säuberung der Wunde, Jodoform, Verband. Anfangs mässige Schmerzen, die bald schwanden, aber Auge blieb injicirt. Deshalb breite Iridectomie nach i. o. Entfernung von möglichst viel Linsensubstanz. Einige Tage nachher bemerkte man, nachdem die Linsenentfernung etwas freieren Einblick in die Tiefe gestattete, gelblichen Schein von i. 0., der doch den Verdacht einer durch Corpus alienum erzeugten eitrig-infectiösen Cyklitis nahelegte; auch geringe Druckempfindlichkeit verstärkte denselben. Lichtschein gut, Projection unsicher. Da cirea 4 Wochen seit der Verletzung der Hornhaut verlaufen waren, so schien die Möglichkeit einer Fortpflanzung des Infectionsprocesses auf das andere, linke Auge, das bislang absolut intact war, nicht ausgeschlossen und so wird von dem gerade anwesenden Vater des Knaben die Erlaubniss zur Enucleation des verletzten Auges erbeten, aber nicht erreicht. Da aber nach weiteren 10 Tagen die Druckempfindlichkeit, sowie der gelbliche Schein aus der Tiefe des rechten Auges zunahm, wird nochmals dringend von dem Vater die Erlaubniss nachgesucht; der Vater erschien nach zwei Tagen, gerade an dem Morgen, als das linke, gesunde Auge die erste Injection und Lichtscheu zeigte, und der Patient über etwas Nebelsehen klagte. Es zeigte sich nun das linke Auge eben merklich injicirt; ophthalmoskopisch Papille, namentlich die innere Hälfte, stark roth, Gefässe ausgedehnt und gesehlängelt, an die Papille sich anschliessend, circa $3 \mathrm{P}$. weit in die Peripherie sich erstreckend, diffuse graue Netzhauttrübung, im Glaskörper keine flottirende Opacität; ob die äussersten, der Netzhaut anliegenden Schichten ganz klar, ist nicht sicher zu entscheiden; Iris von normaler Farbe und Reaction, auf einen Tropfen Atropin völlig gleichmässig rund und maximal erweitert, Kammerwasser klar, aber zahlreiche feinste Beschläge auf der unteren Hälfte der hinteren Hornhautwand. $\mathbf{S}={ }^{20} / 30$; leider also das ausgesprochene Bild der beginnenden "sympathischen Ophthalmie"; die letzten zwoi Tage war der Knabe L. nicht ophthalmoskopirt worden, also nicht festzustellen, wann die Neuroretinitis begonnen: die Beschläge aber hatte er sicher erst seit diesem Morgen, da er den Abend vorher, wenigstens focal, noch angesehen war; 
Zur Pathogenese d. sympath. Ophthalmie (Nachtrag). 333

diese konnten sich also erst während der Nacht eingestellt haben. - Auch jetzt noch wollte der Vater des Knaben die Enucleation anfänglich verweigern und erst nach energischer Aussprache meinerseits und dem eigenen Wunsche des kleinen Patienten selbst, gestattete er dieselbe, die denn auch unverzüglich ausgeführt wurde.

Was nun aber das zweiterkrankte Auge anging, so musste es, meiner Theorie nach, bereits mit Mikroorganismen inficirt sein, ja, ich nahm ohne Weiteres an, dass die Beschläge der Descemeti, bei Abwesenheit eines jeden Zeichens einer Iritis*), von Mikroorganismen herrühren möchten, die durch den Opticus in das Auge eingeführt, vermöge der Lymphbahnen, nicht durch den Uvealtractus langsam vorwärts schreitend - gleich wie ich es beim Kaninchenexperiment gesehen - in die vordere Kammer gelangt und sich hier an der hinteren Hornhautwand festgesetzt hätten. Daraus ergab sich für mich praktisch die Nothwendigkeit, durch Punction der vorderen Kammer den Versuch zu machen, die fraglichen Infectionskeime möglichst $\mathrm{zu}$ entfernen - zugleich aber war die Constatirung von dem Vorhandensein von solchen der Prüfstein für die Richtigkeit meiner ganzen Theorie. So wurde denn nach sorgfältigster Süuberung der Bedeckungen des linken Auges, der Conjunctivalsack mit 2 procentiger Carbollösung, dann mit Sublimat 1:1000 ausgewaschen, mit einer broad needle die Punction der vorderen Kammer ausgeführt und das ausfliessende Kammerwasser, das wie sofortige focale Untersuchung der Comea zeigte, die Beschläge der Descemeti mit enthalten musste, mit einem eben frisch ausgeglühten Tropfröhrchen aufgesogen, sofort auf bereit gehaltenes Fleischinfuspeptonagar im langen Reagensglase übertragen und in den Brut-

*) Erst nach 6 Tagen zwei feine hintere Synechieen, die Atropin löste. 
apparat gebracht. Der geringe Rest, der im Tropfglase verblieb, wurde zur Herstellung von Trockenpräparaten auf Objectglüschen übertragen und mit Gentianaviolett gefärbt. Nach 36 Stunden zeigten sich auf dem Fleischinfusagar die Anfänge einer Colonienbildung in Form zarter, glänzend weisser Fleckchen, die sich bald weiter entwickelten, wie wenn ein Tropfen weisser Oelfarbe ausgestrichen wird, kurz in der allertypischsten Form das ganz charakteristische Bild des weissen Eiterpilzes, des Staphylococcus pyogenes albus (Rosenbach) darboten. Die mikroskopische Untersuchung bestätigte das, und erwies die Cultur gleichzeitig als Reincultur ohne jede Beimengung.

So blieb nur noch übrig, auch die pathogenen Eigenschaften der gezüchteten Cultur zu erweisen. $\mathrm{Zu}$ diesem Zwecke wurden Kaninchen damit in die vordere Kammer, sowie den Glaskörper geimpft; das Resultat war: infectiöse destruirende Entzändung*), wie bei den Versuchen mit anderweitig gezüchteten Eiterpilzen auch.

Die Untersuchung der von dem frisch abgelassenen Kammerwasser mit Beschlägen angefertigten Trockenpräparate, die mit Gentianaviolett gefärbt waren, ergab eine grosse Menge gleichmäsig runder Mikrokoccen, mässig viel Endothelzellen der Membrana Descemeti, verschwindend wenige Lymph- resp. Eiterkörperchen und ein Fibrinnetz; der grösste Theil der Beschläge wurde danach von in Fibrin eingeschlossenen Mikroorganismen dargestellt; kein Wunder also, wenn der Züchtungsversuch eine Reineultur von letzteren ergab. thalmitis.

*) d. h. chronische Iridochorioiditis, nicht acute Panoph- 
Zur Pathogenese d. sympath. Ophthalmie (Nachtrag). 335

Ich habe noch einiges Wenige über die Untersuchung des ersterkrankten, enucleirten Bulbus hinzuzufügen. Es fand sich etwas nach hinten und innen vom Ciliarkörper und zwar nach i. o. ein Steinstück von 3,5 Mm. Länge und stellenweis $2 \mathrm{Mm}$. Dicke, in feine Eiterflocken eingehallt; die Papille pilzförmig geschwollen, mit enorm erweiterten Gefässen und Blutungen, wie eine Stauungspapille. Mikroskopisch: Neuritis optica, papillitis, Aderhaut an der Papille um Vielfaches durch Eiterinfiltration verdickt; auf Quetsch- und Trockenpräparaten aus dem Eiter um das Steinstüek und von der Papille typische Mikrokokken; bei Impfungen auf Fleischinfusagar: Staphylococcus albusEntwickelung um die Impfstückchen.

Ich glaube, dass meine Theorie an diesem paradigmatischen Falle ihre Prüfung gut bestanden hat, dass sie für diese sogenannte "sympathische Ophthalmie" unanfechtbar ist. Denn der Nachweis der Mikroorganismen in den frischen Beschlägen des eben erkrankten zweiten Auges schliesst gleichzeitig den Beweis der Sehnervenleitung in sich, selbst wenn, wie hier, keine Neuroretinitis vorhanden wäre; ich darf hier auf Leber's *) Ausspruch verweisen: „Wenn die sympathische Entzündung eine infectiöse ist, so kann die Fortleitung nur durch den Sehnerven erfolgen."

Ich darf endlich für die practische Verwerthung darauf hinweisen, dass Beschläge auf der Membr. Descemeti in derartigen Fällen kein Zeichen von Iritis zu sein brauchen, sondern bei intactem Uvealtractus, Mikroorganismen-Praecipitate darstellen können, deren Entfernung durch Punction geradezu im Interesse des Kranken verlangt werden muss. Bei meinem Kranken**) besteht die Neuroretinitis noch

*) Dieses Achiv XXVII. 1, p. 331. behandle.

**) Den ich übrigens nebenbei mit kräftiger Innnctionscur 
fort, die Beschläge auf der hintern Hornhautwand haben sich bislang nicht erneuert, Lichtschen und etwas Nebligsehen noch vorhanden, kein neues Zeichen von Iritis. Doch ist die Zeit viel zu kurz, um über den weiteren Verlauf etwas Abschliessendes aussagen zu können; ich hoffe bei späterer Gelegenheit darauf zurückzukommen.

\section{Erklärung der Abbildungen}

zil der Arbeit von Dr. Carl Grahamer: ,Ein Beitrag zur pathologischen Anatomie des Hydrophthalmus congenitus" p. $265-300$.

Fig. 1. Untere Bulbushälfte in doppelter Vergrösserung. a tiefste Furche auf der hinteren Homhantfläche.

Fig. 2, 3, 4, 5, 8. Die verschiedenen Veränderungen im CornealEpithel und in den subepithelialen Schichten; gez. bei Leitz Oeul. I bezw. III und Objectiv 7.

Fig. 6. Cornealcyste. Leitz Oc. III, Objectiv 4.

Fig. 7. Zerklïftung des Corneal-Parenchyms und Ustrrbildung in der Membran. descemet. Leitz Oc. II, Objectiv 4.

Fig. 9 u. 10. Die Veränderungen der Endothel-Lage der Membran. descemet. Leitz Oc. II, Objectiv 7.

Fig. 11. Die Vorderkammerwinkel-Region. Leitz Oc: III, Objectiv 4.

Fig. 12. Flächenpräparat des Ligament. pectinat. Leitz Oc. I, Objectiv 7. 